\title{
Reembolización de arterias uterinas por hemorragia posparto en una segunda gestación
}

\author{
Nuria López V. ${ }^{1}$, Gabriel Vegas G. ${ }^{2}$, Sara Arrieta B. ${ }^{1}$, Carlos Iglesias S. ${ }^{1}$, \\ Antonio González G.2 \\ ${ }^{1}$ Servicio de Obstetricia y Ginecología, ${ }^{2}$ Servicio de Fisiopatología Fetal, Hospital Universitario La Paz, Madrid, España.
}

\section{RESUMEN}

La hemorragia posparto es la principal causa de muerte obstétrica. Presentamos el caso de una gestación y parto normales tras la embolización bilateral de ambas arterias uterinas, por hemorragia posparto en la gestación anterior. La paciente requirió de una nueva embolización bilateral de arterias uterinas en esta segunda gestación con buen resultado obstétrico. La embolización arterial pélvica en el manejo de la hemorragia posparto refractaria al tratamiento médico, permite en ocasiones evitar el tratamiento quirúrgico, conservando la fertilidad de la paciente.

\section{PALABRAS CLAVE: Hemorragia posparto, embolización arterial pelviana, fertilidad}

\section{SUMMARY}

The postpartum hemorrhage is the main cause of obstetric death. We report a case of normal pregnancy and delivery after bilateral embolization of uterine arteries due to postpartum hemorrhage in a previous pregnancy. A new bilateral embolization of uterine arteries was needed in this second pregnancy with good obstetric results. The pelvic arterial embolization in the management of postpartum hemorrhage refractory to medical treatment, avoids the surgical treatment, preserving fertility.

\section{KEY WORDS: Postpartum hemorrhage, pelvic arterial embolization, fertility}

\section{INTRODUCCIÓN}

La hemorragia posparto (HPP) se define como aquel sangrado vaginal mayor a $500 \mathrm{cc}$ tras un parto vaginal o superior a $1000 \mathrm{cc}$ tras una cesárea, o aquella hemorragia que produce una inestabilidad hemodinámica en la paciente o la necesidad de transfusión. Esta complicación se produce en un $4-6 \%$ de los partos y es la primera causa de muerte obstétrica materna directa.

Ante una HPP se recomienda una actuación ordenada intentando controlar las distintas posibles causas del sangrado (1). Debemos revisar el canal blando del parto en busca de lesiones y de persistencia de restos ovulares intrauterinos. La atonía uterina es la causa prevenible más importante de HPP grave. El manejo activo del alumbramiento, especialmente a través del uso de fármacos uterotónicos hace posible controlar el sangrado. Sin embargo, hay casos más graves en los que se produce una coagulopatía que requiere también del uso de hemoderivados para su control. Hasta ahora la alternativa ante el fracaso del tratamiento médico consistía en distintos procedimientos quirúrgicos (ligadura de arterias hipogástricas, métodos de compresión uterina, histerectomía...) que podían 
complicarse en este tipo de pacientes hemodinámicamente inestables (2).

Desde 1979 se ha ido perfeccionando una técnica de radiología intervencionista: la embolización de arterias uterinas que actualmente presenta un grado de recomendación $\mathrm{B}$ con una tasa de éxito estimada entre el $70-100 \%$. Consiste en la cateterización de los vasos sangrantes ocluyéndolos de forma selectiva y distal de tal manera que al disminuir la presión de perfusión, los fenómenos fisiológicos de hemostasia y coagulación detengan el sangrado. Como lo habitual es que ambas arterias uterinas estén implicadas, es frecuente la embolización bilateral y aunque tras este procedimiento parece que se preserva la fertilidad, son pocos los casos publicados de gestaciones posteriores a la embolización arterial selectiva de ambas arterias uterinas.

El objetivo de esta comunicación es presentar un caso de reembolización selectiva de las arterias uterinas en una paciente con una HPP severa.

\section{Caso clínico}

Se describe el caso de una paciente de 36 años cuyo primer embarazo cursó sin incidencias con un crecimiento fetal adecuado. Se indujo el parto a las 40 semanas de gestación por una amnioscopia positiva. El parto evolucionó favorablemente en 6 horas naciendo un recién nacido mujer de 2680 gramos con un $\mathrm{pH}$ posparto arterial de 7.32 y de 7.34 en vena. Se suturó un desgarro de $2^{\circ}$ grado sin complicaciones. El puerperio inmediato tuvo una evolución satisfactoria con una buena involución uterina, siendo dada de alta a las 48 horas. La paciente acudió a la urgencia a los 18 días del parto por una hemorragia espontánea de una hora de evolución sin haber presentado sangrados abundantes en los días previos. Se ingresa a la paciente por abundante hemorragia sin objetivarse lesiones en el canal del parto ni restos ovulares, con buena retracción uterina a la exploración. Los exámenes muestran una anemia leve (hemoglobina: 11,6 g/dl y hematocrito: $34,1 \%$ ) con una coagulación normal. Se indica oxitocina en sueroterapia de mantenimiento. Al día siguiente se realiza una ecografía abdominal y vaginal que muestra un útero bien involucionado con un diámetro longitudinal de $101 \mathrm{~mm}$, sin restos ovulares en su interior. No se apreciaba líquido libre en el espacio de Douglas ni patología anexial. Se indica metilergometrina $0,125 \mathrm{mg}$ cada 8 horas. Ante la disminución del sangrado se otorga el alta con tratamiento oral a las 24 horas del ingreso. Reingresa a las 10 horas del alta por sangrado muy abundante. En la analítica de control presenta una disminución de 5 puntos de hemoglobina (hemoglobina: $6,2 \mathrm{~g} / \mathrm{dl}$ y hematocrito: $17,4 \%$ ) con coagulación normal. Se transfunden 5 concentrados de glóbulos rojos, 6 unidades de plasma fresco congelado y 4 gramos de fibrinógeno. Se realiza revisión del canal del parto y legrado uterino.

La paciente continúa con un sangrado importante que no se consigue controlar, por lo que se decide la embolización de ambas arterias uterinas y de la arteria cérvico vaginal izquierda. Precisa también de la transfusión de otros 6 concentrados de glóbulos rojos y de 240 UI de factor VII recombinante. Durante la transfusión presenta un eritema generalizado y prurito que cede con corticoides y dexclorfeniramina. La evolución posterior es favorable con una analítica al alta con 11,4 g/dl de hemoglobina y $32,2 \%$ de hematocrito.

Tras este embarazo la paciente tiene dos abortos, logrando un embarazo evolutivo al tercer año. Esta segunda gestación tiene una evolución sin incidencias hasta la semana 36 en la que se observa que el crecimiento fetal es dos semanas menor de lo esperado con un estudio de Doppler en arteria umbilical normal. Dadas las características favorables del cérvix, se decide finalizar la gestación a las 37 semanas. El nacimiento se produce mediante un parto vaginal eutócico. Presentando un sangrado posparto abundante. Se revisó el canal blando del parto sin encontrarse lesiones. Se realizó un legrado uterino en el que se obtuvieron escasos restos. Se objetivó una tendencia a la atonía uterina que fue tratada con uterotónicos (misoprostol, oxitocina y metilergometrina) y mediante la transfusión de 3 concentrados de hematíes y 4 gramos de fibrinógeno. Como a pesar del tratamiento persistía el sangrado y dados los antecedentes de la paciente, se realizó una nueva embolización selectiva de ambas arterias uterinas (Figuras 1, 2 y 3), logrando el control de la hemorragia. La paciente fue dada de alta a los tres días del parto en tratamiento con ferroterapia.

\section{DISCUSIÓN}

La embolización selectiva arterial a través de radiología intervencionista logró buenos resultados en el tratamiento de las emergencias hemorrágicas digestivas en los años 70. En el campo ginecoobstétrico la embolización de emergencia de la hemorragia posparto fue por primera vez descrita por Brown y cols en 1979 (3). Doumouchtsis y cols (4), en una revisión sistemática del manejo conservador de la hemorragia posparto sitúa las tasas de éxito de la embolización arterial en el manejo de la HPP en el 90,7\%, del taponamiento con balón 


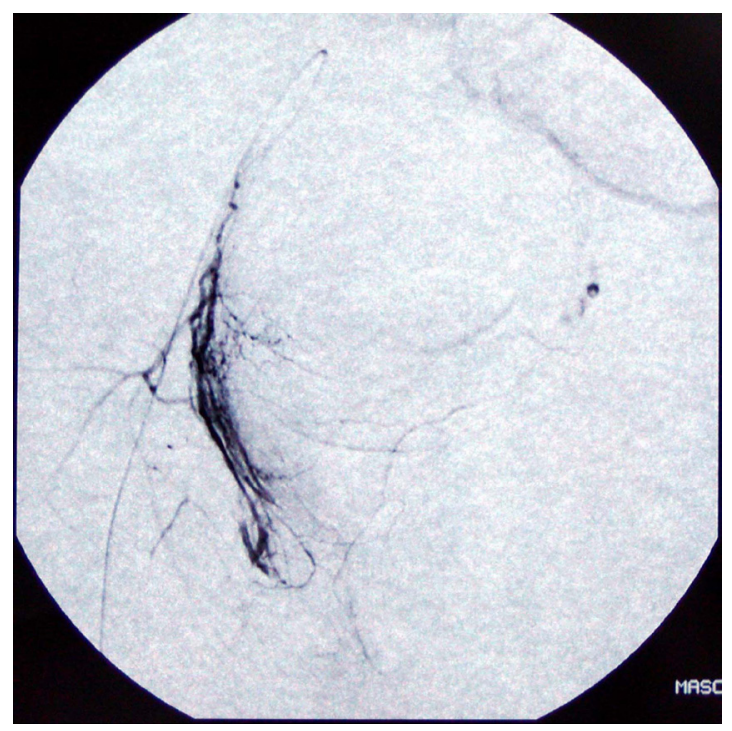

Figura 1. Arteria hipogástrica derecha: extravasación de ramas distales.

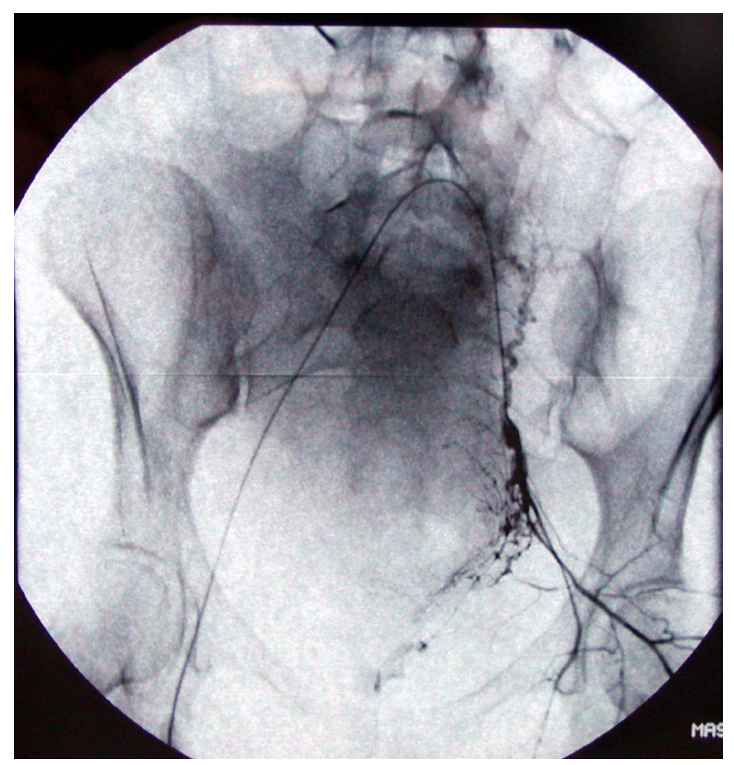

Figura 2. Arteria hipogástrica izquierda: extravasación de ramas distales.

intrauterino en el $84 \%$, para las suturas compresivas del útero en el $91,7 \%$ y para la devascularización pélvica y ligadura de arteria iliaca interna en el $84,6 \%$ sin encontrar diferencias estadísticamente significativas entre los diferentes métodos.

Las principales ventajas que ofrece la embolización uterina son el acceso rápido, la reducción casi

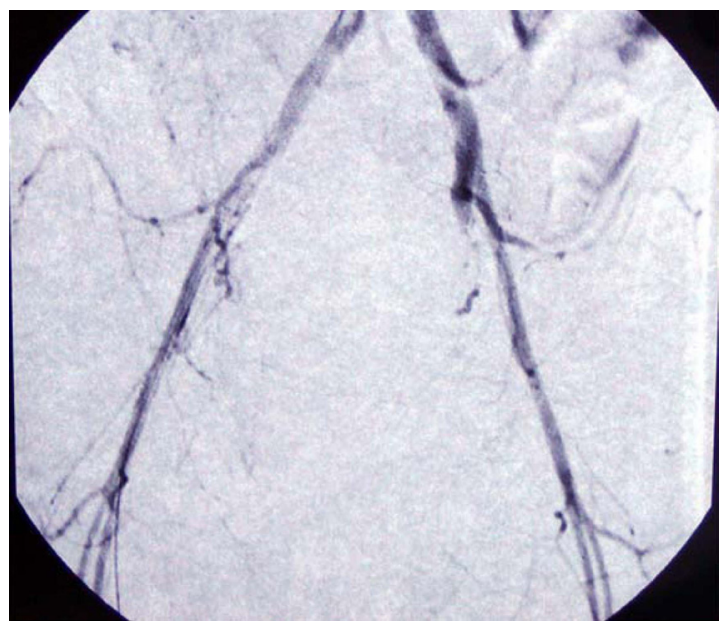

Figura 3. Arteriografía de control posembolización.

inmediata de la hemorragia activa (la mayoría de los estudios observan una reducción marcada del sangrado en las primeras horas) con una oclusión selectiva de los vasos afectados, respetando el resto de la circulación pélvica (5). Se puede detectar el sangrado de vasos con flujo mayor a $1 \mathrm{~mL} / \mathrm{min}$.

El procedimiento se realiza bajo sedación evitando una anestesia general que en estos pacientes hemodinámicamente inestables puede suponer un empeoramiento de su situación clínica. También permite la posibilidad de reembolización, ya sea del mismo territorio, o de eventuales colaterales. Además conserva el útero manteniendo así la fertilidad posterior (6).

Aunque la embolización arterial selectiva es un método efectivo para tratar la hemorragia obstétrica, son pocos los datos que se conocen sobre las consecuencias en la fertilidad posterior. Sabemos que la oclusión arterial con partículas de alcohol polivinílico (PAV) o microesferas $(700 \mu \mathrm{g})$ no es permanente, permitiendo la recanalización de las arterias en unas 4 a 5 semanas. Sin embargo, el uso de PAV se ha asociado con una mayor frecuencia de necrosis uterina (7).

Son pocas las revisiones que encontramos en la literatura, que recojan casos de gestaciones posteriores a un procedimiento de embolización debido a una HPP. Probablemente este hecho esté en relación con la afectación psicológica de estas pacientes que padecieron esta grave complicación obstétrica. En una de las revisiones más largas de Nizard y cols (8), el $25 \%$ de las pacientes que habían sido sometidas a una embolización arterial selectiva de causa obstétrica, tuvieron una gesta- 
ción posterior y de ellas el $70 \%$ de las gestaciones fueron a término. Refieren que las nuevas gestaciones presentaron un crecimiento fetal adecuado y ninguna paciente requirió de una embolización en esta segunda gestación.

Se ha empleado este procedimiento en casos de fibromas o malformaciones uterinas con un mayor número de gestaciones posteriores (9). En la mayor parte de los casos publicados, en las gestaciones posteriores, el crecimiento fetal era acorde y no se observaban signos de afectación de la circulación úteroplacentaria (10). La reacción secundaria más frecuente es el síndrome postembolización, caracterizado por dolor, náuseas, fiebre y leucocitosis.

La tasa de complicaciones es del 6-7\%. Pueden producirse hematomas, falsos aneurismas de la zona de punción, reacción al contraste, disección arterial, abscesos, necrosis uterina o fístulas vaginales, siendo la más común la fiebre que suele resolverse en 2-3 días.

\section{CONCLUSIÓN}

La embolización de las arterias uterinas es un procedimiento seguro, controla el sangrado y estabiliza a las pacientes hemodinámicamente con un buen resultado técnico y clínico (11); es selectiva y permite conservar la fertilidad de la mayoría de las pacientes, con crecimiento fetal no afectado.

\section{REFERENCIAS}

1. Vegas G, Illescas T, Muñoz M, Pérez Piñar A. Elective pelvic arterial embolization in the management of obstetric hemorrhage. Eur J Obstet Gynecol Reprod Biol 2006;127:68-72.

2. Ornan D, White R, Pollak J, Tal M. Pelvic embolization for intractable postpartum hemorrhage: long - term follow-up and implications for fertility. Obstet Gynecol 2003;102:904-10.

3. Brown BJ, Heaston DK, Poulson AM, Gabert HA, Mineau DE, Miller FJ. Uncontrollable postpartum bleeding: a new approach to hemostasis through angiographic arterial embolization. Obstet Gynecol 1979;54:361-5.

4. Doumouchtsis SK, Papageorghiou AT, Arulkumaran S. Systematic review of conservative management of postpartum hemorrhage: what to do when medical treatment fails. Obstet Gynecol Surv 2007;62:540-7.

5. Hunter LA. Exploring the role of uterine artery embolization in the management of postpartum hemorrhage. J Perinat Neonatal Nurs 2010;24:207-14.

6. Salomon LJ, Tayrac R, Castaigne- Meary V, Audibert F, Musset D, Giorascu R, et al. Fertility and pregnancy outcome following pelvic arterial embolization for severe postpartum haemorrhage. A cohort study. Hum Reprod 2003;18:849-52.

7. Pelage JP, Laurent A, Wassef M, Bonneau M, Germain D, Rymer R, Flaud P, Martal J, Merland JJ. Uterine artery embolization in sheep: comparison of acute effects with polyvinyl alcohol particles and calibrated microspheres. Radiology 2002;224:436-45.

8. Nizard J, Barrinque L, Frydman R, Fernández H. Fertility and pregnanacy outcomes following hypogastric artery ligation for severe postpartum haemorrhage. Hum Reprod 2003;18:844-8.

9. Quevedo E, Tan J, Araujo G, Cueva M, Fernández I. Embolización uterina de emergencia en la hemorragia masiva. Rev Peruana Radiol 2003;5:34-7.

10. Descargues G, Mauger Tinlot F, Douvrin F, Clavier E, Lemoine J, Marpeau L, Menses fertility and pregnancy after arterial embolization for the control of postpartum hemorrhage. Hum Reprod 2004;19:339-43.

11. Touboul C, Badiou W, Saada J, Pelage JP, Payen $\mathrm{D}$, Vicaut $\mathrm{E}$, et al. Efficacy of selective arterial embolization for the treatment of life-threatening postpartum haemorrhage in a large population. PLoS One 2008;3:e3819. 